\title{
The Role of Pediatric Trauma Centers in Disaster Preparation
}

Katie W. Russell, $M D^{1, *}$

Stephen J. Fenton, $M D^{1}$

Erik G. Pearson, $M D^{2}$

\author{
Address \\ ${ }^{*}, 1$ Primary Children's Hospital, 100 N Mario Capecchi Drive, Suite 3800, Salt Lake \\ City, UT, 84113, USA \\ Email: katie.russell@hsc.utah.edu \\ ${ }^{2}$ Sunrise Children's Hospital, Las Vegas, USA
}

Published online: 16 May 2019

(C) Springer Nature Switzerland AG 2019

This article is part of the Topical Collection on Disaster Medicine

Keywords Disaster preparedness - Las Vegas shooting - Pediatric · Public education

\begin{abstract}
Purpose of review Disasters, both natural and manmade, are becoming more common. Children often compose up to $30 \%$ of those individuals injured in a disaster. The level of preparedness for a surge of pediatric patients after a disaster is variable.

Recent findings Pediatric trauma centers need to be able to take care of multiple children and adults and assist other centers with the care of children. A disaster plan with associated training should be instituted at all hospitals. Collaboration across multiple facilities and agencies is essential in planning. Additionally, it is the duty of medical professionals to train the public in disaster preparedness.

Summary Learning through the experiences of others is perhaps the most powerful means of education. A case study of how one hospital handled the deadliest shooting in US history can teach us many lessons.
\end{abstract}

\section{Introduction}

Health care facilities and personnel should be prepared to treat patients who are injured in a disaster. The United States Federal Emergency Management Agency (FEMA) defines a disaster as "an occurrence that has resulted in property damage, death(s), or injuries to the community" $[1 \bullet \bullet]$. These occurrences may be a result of natural catastrophes (hurricanes, earthquakes, wild fires, etc.) or man-made mass casualty incidents (MCI) defined by the World Health Organization (WHO) as "an event which generates more patients at one time 
than locally available resources can manage using routine procedures" (terrorist attacks, mass shootings, airplane crash, building collapse, etc.) [2].

Natural catastrophes are becoming an ever more common occurrence within the USA and throughout the world. The website OurWorldInData.org, a collaboration between the Oxford Martin Programme on Global Development and the non-profit organization Global Change Data Lab, publishes data demonstrating how global living conditions and the earth's environment have changed since 1900 . Their data demonstrate a marked increase in natural catastrophes over this period of time (Fig. 1). They also show that the death rate per 100,000 people from these disasters has decreased in the same period of time with a tremendous global increase in the total damage costs [3].

Unfortunately, mass casualty incidents have also become the new normal, not only in the USA, but globally as well. These take the form of mass shootings, acts of terrorism, accidents involving busses, trains, or airplanes, as well as other tragic events such as fires, explosions, or structural failures where a large number of people are present. The Global Terrorism Index Score increased ninefold from 2000 to 2015. In the USA, mass shootings have become a national epidemic. Recently, the National Center for Victims of Crime reported that the number of mass shootings that occurred over the last 10 years is 2.4 times greater than the previous decade. They also state that $57 \%$ of all recorded mass shootings occurred within the past 10 years [4].
Upon reviewing 8303 patients involved in a disaster or major emergency incident over a 25 -year period of time, Reilly et al. demonstrated that nearly $2 / 3$ of these patients were transported to medical facilities by means other than an ambulance, the great majority of which were transported by themselves [5]. Other studies have demonstrated similar findings, mainly, patients find their own transportation and are typically taken to hospitals closest to the event regardless of the type of medical facility $[6,7]$. Even when disaster protocols exist to aid in the distribution of patients to local and regional hospitals in an attempt to avoid overwhelming such facilities, these protocols do not always function as anticipated when applied to real world events [2]. Therefore, all facilities (adult, adult with pediatric capabilities or free-standing pediatric) should have disaster plans that prepare and help guide treatment of both adult and pediatric patients following a disaster. Additionally, adult centers which do not typically treat pediatric patients need to include in their plans how to address the unique staffing characteristics and equipment needs required to treat injured children $[1 \bullet \bullet]$.

Approximately, $10-30 \%$ of patients in a mass casualty incident are children [8]. It is crucial that pediatric trauma centers (PTC) be prepared for such disasters due to the high probability that those hospitals and their providers will be involved in some capacity [9]. The purpose of this review is to discuss the capabilities of a PTC and its role in disaster preparation. Central to this discussion is the experience of Dr. Erik G. Pearson, a

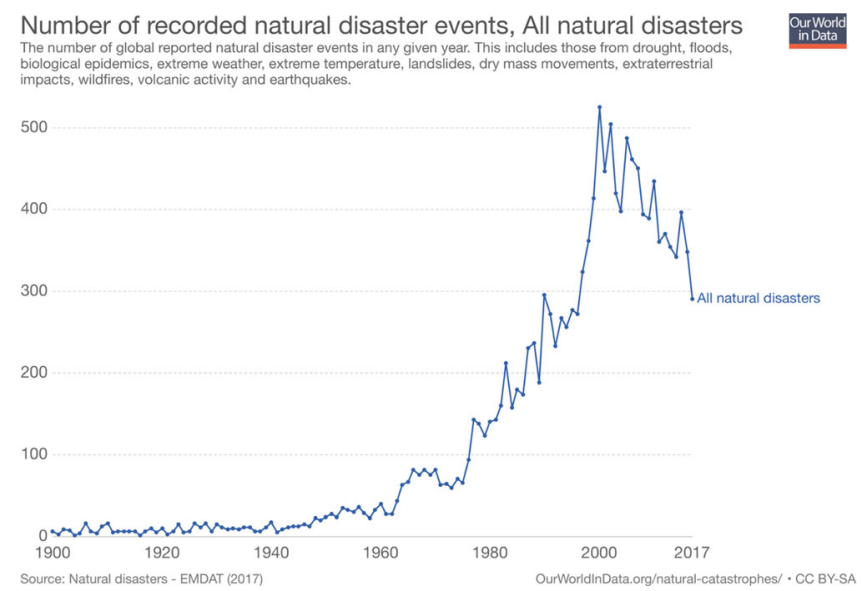

Fig. 1. Number of recorded natural disaster events. All natural disasters 
pediatric surgeon, who participated in the medical response at Sunrise Hospital and Medical Center (https:// sunrisehospital.com) to the Las Vegas shooting which occurred during the Route 91 Harvest Musical Festival on October 1, 2017 resulting in the death of 58 people and injury of an additional 869 . Upon reading this paper, the reader should gain further insight into how to better prepare for disaster response.

\section{Role of the pediatric trauma center}

There are three likely scenarios that will require the expertise of a PTC. First, children involved in a disaster may be transported directly to such a center for care. While this scenario may present the most logical pathway, history has shown that this does not always happen during actual events [2]. Second, because PTCs are less common than adult facilities, it is more likely that children will be initially taken to the closest hospital, then, subsequently require further assistance from pediatric subspecialists, necessitating transfer to a PTC. Finally, based on proximity to the event, adult patients may be immediately delivered directly to a PTC for care. Each of these scenarios presents a complex set of factors that PTCs must prepare and train for to insure a proper disaster response. Additionally, pediatric patients are smaller in size and stature, require specialized equipment and, at times, present with different physiologic trends. These traits can lead to worse outcomes for these patients when cared for by practitioners that are more familiar with the adult patient population and lack the expertise and resources to care for injured children [10•]. As pediatric providers, it is our job to not only train ourselves, but to also help prepare our non-pediatric colleagues. This task begins with our personal preparation but must also extend beyond our institutions and regional systems to the general public as well so that we are all best prepared for whichever disaster scenario presents itself.

Although no perfect method of preparing for an unknown disaster exists, there are generalized practices that can be gleaned from previous event responses. At present, US hospital preparedness for disasters involving pediatric patients is highly variable [11, 12]. A multitude of resources are available, through agencies such as Emergency Medical Services for Children, that can aid in both the implementation or update of a preparedness plan. While didactic education can be an initial step, experiential activities provide increased learner involvement, increased buy-in, and longer knowledge retention. Simulation has been successful in many formats such as table-top exercises, virtual reality, mannequin utilization, and actor's role-playing standardized patients. In situ drills are the gold standard [10•]. These drills improve practice standards, communication, resource utilization, and teamwork but also expose situational and systemic weaknesses that require improvement and can be targeted during future practice sessions. We recognize that it is difficult to mandate participation in disaster drills to already busy staff, but multiple studies have highlighted this method as the most effective means of relevant preparation. It is also important to repeat drills to help improve effectiveness $[13,14,15 \bullet]$. We encourage readers to start with 
small, realistic, and manageable trainings before progression to larger, multidisciplinary or multi-institutional scenarios.

Institutional emergency planning and corresponding drills are a vital initial step before transitioning to a more robust multi-institutional and multi-agency simulation [16]. Of the scenarios presented earlier, the most likely is a surge of injured children delivered to a non-pediatric center. Simulation of such a disaster could greatly assist an actual response by local hospitals, EMS agencies, and allied health professionals. One solution is to mobilize and transport a pre-determined group of pediatric specialists to the receiving institution instead of transferring many critically-ill children to a pediatric facility. These specialists can aid in triage, treatment, and transport decisions. Additionally, as needed telemedicine technology could also be utilized in this scenario to connect pediatric experts with distant providers to assist in evaluation of the patients and care management decisions. In addition to training, it is paramount that an emergency stock of pediatric specific supplies be stored and available for use during a disaster. This equipment needs to be inventoried regularly to assure currency, utility, and functionality for mass casualty incidents involving children.

One of the most important aspects in disaster preparedness is public education. All trauma centers can and should participate in this facet of training. Bystanders and survivors often are forced into first responder roles when tragedy strikes. Who better to train this population than those of us that treat trauma on a daily basis? The current "Stop the Bleed" educational campaign is a poignant example of such an undertaking. "Stop the Bleed" was pioneered by the American College of Surgeons and other agencies to teach the general public about basic bleeding control techniques that save lives. The driving force behind and success of this campaign have been the need for these skills, along with CPR training, in a society where violent crime has become so prevalent $[17,18]$.

\section{Walking through a disaster: the Las Vegas mass casualty incident}

Mass casualty incidents strike the heart of communities and stress the healthcare system from first responders through trauma triage and the multiple specialties called to care for victims and their families in the days, weeks, and months that follow. Hospitals, those caring for both children and adults, need to be prepared with both knowledgeable and effective staff as well as the materials required to manage these events. Importantly, whether a hospital is a level 1 trauma facility or a community hospital that rarely sees trauma, it may be the first point of contact following a mass casualty incident. On the evening of October 1, 2017, a lone gunman opened fire into a crowd of thousands at the Route 91 Harvest music festival in what would be the largest mass shooting event in US history. In the moments that followed the initial gunshots from the 32nd floor of the Mandalay Bay hotel, first responders reacted quickly from the scene and locally to triage. In those moments, the message on the police radios "Automatic fire...country music concert" alerted local emergency rooms and emergency physicians that a wave of gravely injured patients would be arriving and they would have just minutes to prepare.

Multiple hospitals in the Las Vegas valley, trauma centers, and non-trauma centers began receiving the first patients shortly after 10 p.m. on that Sunday evening. In total, approximately 620 patients were treated at 13 hospitals citywide 
[19••]. Sunrise Hospital, a level 2 trauma hospital located close to the Las Vegas strip, received the greatest number of patients following the mass shooting. The majority of victims arrived by private vehicle, coming by pairs and groups in cars, the backseats of taxis, and crowded in the beds of pickup trucks. Many victims also came to the ambulance bay with first responders. Few were dead; many were dying, and all needed quick and effective medical attention.

Dr. Kevin Menes, an emergency room physician at Sunrise hospital, was on duty the night of the shooting. After hearing the police scanners, Dr. Menes set a plan into motion to save many lives that night and in the days that followed. Dr. Menes stressed several key points for the triage team, physicians, hospital staff, and the hospital administration to consider when confronted with a mass casualty incident and the immediate influx of a high volume of critically injured patients [20]. First is to plan ahead. For the triage team, this means knowing who will be the team lead, where the patients will be treated, what clinical choke points to expect, how teams will communicate and how to effectively triage patients and provide life-saving care. This also means frequent drilling for patient surge and mass casualty incidents by all levels of the team. For the hospital, this means activating "code triage" and the Hospital Incident Command Systems (HICS). "Code Triage" at Sunrise Hospital and other hospitals is an emergency preparedness plan that alerts all physicians and staff to remain on site, initiates preparation for a patient surge, and activates HICS. After activating "Code Triage" at Sunrise Hospital, every available gurney was brought to the emergency department; the emergency room was cleared of patients; non-critical patients were moved from the intensive care units; the wards were prepared for the incoming injured and all available physicians; surgeons and staff were called in to help.

Patients begin to arrive and after activation of the hospital's disaster response plan, the administration and the triage team need to be aware of choke points that will occur on both clinical and systematic fronts. Clinical choke points include congested patient flow, drug and blood availability, imaging, and supplies. The initial clinic choke point identified by Dr. Menes was patient flow into and out of the emergency department. He identified the solution to be effective and efficient triage, designated resuscitation areas, and finding a goal destination for every patient. As soon as patients arrived, each had bilateral large bore intravenous catheters placed and were triaged in the ambulance bay according to severity of injury. Patients were sent to "stations" in the emergency room for resuscitation by trauma surgeons and emergency physicians based on their severity of injury. The most severe life-threatening injuries were triaged as red and sent to station 1 in the trauma bay closest to the operating rooms. Patients with severe life-threatening injuries but were expected to survive the next hour were triaged orange and sent to station 2. Patients with torso and neck injuries or proximal extremity injuries and were expected to survive past the hour were triaged yellow and sent to station 4 . Finally, the walking wounded, patients with minor injuries were triaged green and placed in the rapid track area. This organized system of grouping patients according to severity saved time and helped facilitate recognition of injury severity and rapid resuscitation by other providers. An important point to be remembered in the triage of a mass casualty incident is that triage is a dynamic process. Patient triaged to orange may quickly progress to red and some yellow patients will become orange as their injury evolves, especially considering the immense energy of injury from high velocity semi-automatic weapons like those used in the Las Vegas mass casualty incident. 
With multiple gunshot wound patients arriving every minute, flow meant moving the critical life-threatening injuries out of the "station 1" trauma bay and into the operating rooms as soon as possible. Patients with immediate surgical need were taken directly to the operating room while patients with significant injuries were resuscitated in the emergency room. Two groups of patients were identified. Patients with injuries to the torso requiring thoracostomy tubes and endotracheal intubation as well as patients with head injuries requiring intubation began to restrict space in the emergency room and limit flow. Dr. Stephanie Davidson, a pediatric cardiac anesthesiologist, worked with the intensivists to establish secondary triage for these patients in the intensive care units (ICU). Patients who were hemodynamically stable after initial resuscitation with torso injuries were intubated, and thoracostomy tubes were placed if needed, and the patients were taken to the cardiovascular ICU where a trauma surgeon, anesthesiologist, and intensivist continued resuscitation and secondary triage. If the patient became hemodynamically unstable or was determined to need immediate surgical intervention, they were taken to the operating room. Patients with head injuries were intubated and taken to the neuro-ICU, and another team of surgeons, anesthesiologists, and intensivists continued their resuscitation. This spirit of innovation and creation of secondary triage freed up the emergency room and led to rapid transit of patients from the ambulance bay, through the specific triage stations in the ER and on to the operating room for surgery or to the ICU for secondary triage allowing the prompt care for the waves of patients that continued through the night.

The next series of clinical choke points included limitations in the ability to provide direct care to patients. In order to speed up the delivery of medications for intubation and pain management, the Pyxis machines were disengaged and nurses were able to obtain and deliver drugs more efficiently. Emergency department pharmacy technicians were specifically designated to ensure medication availability. The blood bank ensured the shifting of supplies, and nurses were given units emergency release blood so it could be more easily distributed to patients requiring immediate transfusion. Nearly 500 blood components were transfused, and the overall RBC-to-plasma-to-platelet ratio was 1.0:0.54:0.81 [21]. Additionally, nurses were assigned one-to-one with patients to ensure attentive, safe, and continuous care. Radiologists were paired with imaging technologists so that reads for portable imaging could be given to the resuscitating physician immediately. As many patients required intubation, ventilators became a scarce resource; therefore, some patients were paired with another similarly size patient on a single ventilator using split tubing. Respiratory therapists were specifically assigned to ventilator support and supply pack creation. To assist with supply chain management, a designated area of the emergency department was created as a staging area to assist with resupplying treatment areas. Todd Sklamberg, the chief executive officer of Sunrise Hospital, was present in the emergency department throughout the night working to make sure that the clinicians and staff had the required resources (supplies and personnel) needed to care for the waves of patients. Creativity, innovation, and persistence provided solutions to many of the clinical choke points that challenged the team on October 1, 2017.

Systematic choke points experienced during this disaster included patient registration, documentation, communication, and coordination. Each of these challenges provided important lessons for the healthcare team. With waves of 
patients presenting in large numbers, the patient registration system was easily overwhelmed. Many patients presented without identification, and linking patients with concerned family and friends became challenging. Documentation was difficult through the evening as patients moved rapidly from the ambulance bay through their assigned triage station in the emergency department and on to the operating room, the ICU, or the hospital ward. Deceased patients needed to be placed in a cooled environment despite the hospital morgue being full. As in other mass casualty events, specifically the Boston Marathon bombing (2013), the cellular service crashed making communication outside of the hospital system very difficult. Each of these challenges was met with a solution, either during the evolution of the MCI or in the post analysis. The patient registration system was transitioned to use the National Hurricane Center naming system to avoid duplicate names for future MCI and patient surge responses. For documentation, a dump bag has been introduced that can be positioned at the foot of each patient's gurney in which all important documents can be placed for subsequent review and to help complete appropriate charting once the event situation has stabilized. At Sunrise hospital, the endoscopy suites can be individually cooled to the appropriate temperature and this area acted as a temporary morgue. Some specialized operating rooms, i.e., cardiac, also have similar capabilities. An internal hospital cellular system communicating within the Wi-Fi network stayed operational, and therefore provider cellular phones were switched into Wi-Fi assist mode, allowing them to continue to make calls.

As the dawn emerged, patients, family members, and friends required mental health support; in addition, health care providers and hospital staff also needed similar support. The Sunrise Hospital mental health support team was utilized for patient and family support while the Veterans Administration mental health services provided care to physicians and hospital staff. Finally, the environmental services staff was dedicated to all spaces in the hospital making sure that they were safe and cleaned for continued care.

At the end of the night, over 200 patients were treated, 83 surgeries performed and over 100 physicians and 200 nurses responded. As in all mass casualty incidents, many lessons were learned through the evening and in the days, weeks, and months that followed. Preparation and practice with complex events in previous years, strong physician leadership, nursing engagement, support from the residency program, and hospital incident command laid the groundwork for many successes following the mass shooting. In the future, these lessons will hopefully help other healthcare systems prepare for these unwanted events in their own community.

\section{Recommendations}

- Pediatric trauma centers need to be prepared to take care of children and adults and provide aid to those not as familiar with taking care of injured children

- Consider clinical choke points, specifically patient flow following a mass casualty incident and how patients will get from ambulance bay to a 
destination (operating room, intensive care unit, floor, or discharge)

- Secondary triage in the intensive care unit can create valuable space in a crowded emergency department following patient surge

- Systematic choke points including patient registration, identification, and documentation can easily overwhelm existing hospital infrastructure

- Supply chain management is a critical feature to success following a MCI

- Practice disaster scenarios individually, as an institution and as regional systems

- Debrief drills and improve upon systemic weaknesses in subsequent drills

- Everyone must practice because it is unpredictable where the first point of contact may be

- Public education in disaster management is necessary and effective

- A coordinated mental health plan for patients and families as well as healthcare providers and hospital staff considering the effect of second victim phenomena following mass casualty incidents

\section{Compliance with Ethical Standards}

Conflict of Interest

Katie W. Russell declares no conflict of interest. Stephen J. Fenton declares that he has no conflict of interest. Erik G. Pearson declares that he has no conflict of interest.

Human and Animal Rights and Informed Consent

This article does not contain any studies with human or animal subjects performed by any of the authors.

\section{References and Recommended Reading}

Papers of particular interest, published recently, have been

highlighted as:

- Of importance

$\bullet$ Of major importance

1.• Lozon MM, Bradin S. Pediatric disaster preparedness. Pediatr Clin North Am. 2018;65:1205-20 This review discusses the specific differences between children and adults in terms of physical and psychological characteristics. They discuss the Simple Triage and Rapid Treatment (START) triage system and how it was amended to JumpSTART to better triage young children in a mass casualty incident. The authors describe specific techniques for possible threats including: anthrax, plague, small pox, botulinum toxin, chemical agents, and a pandemic inflenza.

2. Postma ILE, Weel H, Heetveld MJ, van der Zande I, Bijlsma TS, Bloemers FW, et al. Patient distribution in a mass casualty event of an airplane crash. Injury. 2013;44:1574-8.

3. Ritchie H, Roser M. Natural catasrophes OurWorldInData.org: 2018 University of Oxford; 2018
[Available from: https://ourworldindata.org/naturalcatastrophes. Accessed November 1, 2018.

4. National Center for Victims of Crime in partnership with the Office for Victims of Crime (2018). NCVRW Resource Guide: Mass Casualty Shootings Fact Sheet; 2018. p. 1-2.

5. Reilly MJ, Markenson D. Hospital referral patterns: how emergency medical care is accessed in a disaster. Disaster Med Public Health Prep. 2010;4:226-31.

6. Carles M, Levraut J, Gonzalez JF, Valli F, Bornard L, 16 authors AFLOAIAITA. Mass casualty events and health organization: terrorist attack in Nice. The Lancet. 2016;388:2349-50.

7. Haas H, Fernandez A, Bréaud J, Dupont A, Tran A, Solla F. Terrorist attack in Nice: the central role of a children's hospital. The Lancet. 2017;389:1007. 
8. Gnauck KA, Nufer KE, LaValley JM, Crandall CS, Craig FW, Wilson-Ramirez GB. Do pediatric and adult disaster victims differ? A descriptive analysis of clinical encounters from four natural disaster DMAT deployments. Prehosp Disaster Med. 2007;22:67-73.

9. Cicero MX, Baum CR. Pediatric Disaster Preparedness. Pediatric Emergency Care. 2008;24:478-81.

10. Bank I, Plotnick LH. A call to action: attention to pediatric-specific disaster preparedness. Arch Dis Child archdischild. 2018;315:461-2 This is a short editorial that is well-written and calls in action improved pediatric disaster preparedness.

11. Ketterhagen TM, Dahl-Grove DL, McKee MR. National survey of institutional pediatric disaster preparedness. Am J Disaster Med. 2018;13:153-60.

12. Chokshi NK, Behar S, Nager AL, Dorey F, Upperman JS. Disaster management among pediatric surgeons: preparedness, training and involvement. Am J Disaster Med. 2008;3:5-14.

13. Toida C, Muguruma T, Hashimoto K. Hospitals' preparedness to treat pediatric patients during mass casualty incidents. Disaster Med Public Health Prep. 2018;58:1-4.

14. Roppolo LP, Pepe PE, Campbell L, Ohman K, Kulkarni $\mathrm{H}$, Miller R, et al. Prospective, randomized trial of the effectiveness and retention of 30-min layperson training for cardiopulmonary resuscitation and automated external defibrillators: The American Airlines Study. Resuscitation. 2007;74:276-85.

15. Gowing JR, Walker KN, Elmer SL, Cummings EA. Disaster preparedness among health professionals and support staff: what is effective? An Integrative Literature Review. Prehosp Disaster Med. 2017;32:321-.

This is a literature review of 36 papers on disaster preparedness concludes that we are likely underprepared for disasters. While the best method of preparation remains unknown, any kind of preparation is better than none. The authors call for improved research as we go forward. They also emphasize training the healthcare team as a whole, including support staff and allied health professionals. Finally, they recommend evaluation of actual disasters in order to evaluate preparedness and better prepare for the next event.

16. Burke RV, Kim TY, Bachman SL, Iverson EI, Berg BM. Using mixed methods to assess pediatric disaster preparedness in the hospital setting. Prehosp Disaster Med. 2014;29:569-75.

17. Ross EM, Redman TT, Mapp JG, Brown DJ, Tanaka K, Cooley CW, et al. Stop the bleed: the effect of hemorrhage control education on laypersons' willingness to respond during a traumatic medical emergency. Prehosp Disaster Med. 2018;33:127-32.

18. AlSabah S, Haddad Al E, AlSaleh F. Stop the bleed campaign_ A qualitative study from our experience from the middle east. Annals of Medicine and Surgery. 2018;36:67-70.

19.• Federal Emergency Management Agency. 1 October After-Action Report; 2018. p. 1-61. The Federal Emergency Management Agency (FEMA) created a report after the Las Vegas Shooting. The report discusses 72 observations and recommendations about the incidence response. They emphasize the importance of preparation and interagency communication

20. Menes K. How one Las Vegas ED saved hundreds of lives after the worst mass shooting in U.S. history. 2010.

21. Lozada MJ, Cai S, Li M, Davidson SL, Nix J, Ramsey G. The Las Vegas mass shooting. J Trauma Acute Care Surg. 2019;86:128-33.

\section{Publisher's Note}

Springer Nature remains neutral with regard to jurisdictional claims in published maps and institutional affiliations. 